\title{
Chronic Non-specific Pain After Gastric Bypass
}

\author{
A. Hussain ${ }^{1} \cdot$ S. El-Hasani ${ }^{2}$
}

Published online: 30 September 2015

(C) Springer Science+Business Media New York 2015

\section{Dear Editor:}

With great interest, we read the article regarding single anastomosis gastric bypass [1]. We congratulate the authors for the fantastic results they have achieved in this particular type of bariatric surgery.

We have experience of over 2,000 Roux-en-Y gastric bypasses and under 200 one-anastomosis gastric bypasses.

We agree with the content and the conclusion of the article, and we would like to compliment the scientific value of it by sharing with you some of our observations on this procedure.

Very occasionally, following Roux-en-Y gastric bypass, we are faced with a scenario that a patient comes with chronic non-specific abdominal pain which we investigate by gastroscopy, ultrasound scan of the gallbladder, CT scan of the abdomen and pelvis, and a diagnostic laparoscopy, and no cause is found for it. Consequently, we explain to these patients and ask them to accept symptomatic treatment.

Bearing in mind the rare occasions that we have dealt in the past [after Roux-en-Y gastric bypass] with problems of jejuno-jejunal anastomosis like perforated ulcer at this anastomosis, intussusceptions, twist of the anastomosis or stricture, we think that these cases of chronic abdominal pain are related to this jejuno-jejunal anastomosis, whether anatomically or

A. Hussain

azahrahussain@yahoo.com

Brighton and Sussex University Hospital, Brighton, UK

2 King's College London, London, UK physiologically, and there are limited diagnostic ways where jejuno-jejunal anastomosis can be investigated.

With our initial experience with a single-anastomosis bypass and our reading of the literatures and most recently your article, we find that this problem of chronic abdominal pain has not been encountered or mentioned and we are optimistic that the single-anastomosis gastric bypass will eliminate not only the problems of internal hernia but also the early and late possible morbidities with the jejuno-jejunal anastomosis one of which we believe is the chronic non-specific abdominal pain and its costly and unyielding investigations.

Regards, Mr. A Hussain and Mr. Shamsi El-Hasani

\section{Compliance with Ethical Standards}

Conflict of Interest The authors declare that they have no competing interests.

Ethical Approval For this type of study, formal consent is not required.

Informed Consent Informed consent does not apply.

\section{Reference}

1. Chevallier JM, Arman GA, Guenzi M, et al. One thousand single anastomosis (omega loop) gastric bypasses to treat morbid obesity in a 7-year period: outcomes show few complications and good efficacy. Obes Surg. 2015;25(6):951-8. 